\title{
$\underline{2}$ АВТОМАТИЧНІ ТА АВТОМАТИЗОВАНІ СИСТЕМИ УПРАВЛІННЯ ТЕХНОЛОГІЧНИМИ ПРОЦЕСАМИ
}

References

1. Axelrod R. The Structure of Decision: Cognitive Maps of Political Elites [Text] / R. Axelrod. - Princeton. University Press. - 1976 - 404 r.;

2. Spravochno-ynormatsyonny portal. [Elektronny resurs] - Rezhym dostupa: www.dic.academic.ru

3. Tsybul'skyy, V. R. Kohnytolohyya. Osnovnye ponyatyya kohnytyvnoho upravlenyya [Tekst] / V. R. Tsybul'skyy, V. V. Fomyn. - Vestnyk kybernetyky. Vip. 1.- Tyumen': Yzd-vo YPOS SO RAN. - 2002. - S. 34 - 37;

4. Raevneva, E. V. Kohnytyvnoe modelyrovanye dlya reshenyya zadach upravlenyya slabostrukturyrovannymy systemamy (sytuatsyyamy) [Tekst] / E. V. Raevneva, N. M. Berest. - Byznesynform. - 2010. - \#5 (2). -S. 40 -43.;

5. Maksymov, V. Y. Kohnytyvne tekhnolohyy dlya podderzhky prynyatyya upravlencheskykh reshenyy [Elektronny resurs] / V. Y. Maksymov, E. K. Kornoushenko, S. V. Kachaev. - Rezhym dostupa: http://emag.iis.ru/arc/infosoc/emag.nsf/BPA/092aa276c601a997c32568c0003ab839.;

6. Aref"yeva, O. V. Ekonomichna stiykist' pidpryyemstva: sutnist', skladovi ta zakhody yiyi zabezpechennya [Tekst] / O. V. Aref"yeva, D. M. Horodyns'ka. - Aktual'ni problemy ekonomiky. - 2008. - \# 8. - S. 83 - 90.;

7. Kulynych, A. A. Antolohyya yntellektual'nkh moduley. [Elektronny resur] - Rezhym dostupa: http://www.raai.org/razrabotki/rgupiim/imlist.html.;

8. Ladanyuk, A.P. Innovatsiyni tekhnolohiyi $\mathrm{v}$ upravlinni skladnymy biotekhnolohichnymy ob"yektamy ahropromyslovoho kompleksu [Tekst] : monohrafiya / Ladanyuk A. P., Reshetyuk V. M., Kyshen'ko V. D., Smityukh Ya. V.. - Kyyiv : Tsentr uchb. lit., 2014. - 279 s.

\section{АДАПТИВНАЯ СИСТЕМА УПРАВЛЕНИЯ ЭЛЕКТРОПРИВОДОМ КОМПРЕССОРА}

\author{
Букарос А.Ю. ${ }^{1}$, Ромчук Н.О. ${ }^{1}$, Букарос В.Н. ${ }^{2}$ \\ ${ }^{1}$ Одесская национальная академия пищевых технологий, Одесса \\ 2 Училище №3 Национального университета ОЮА, Одесса
}

Copyright (C) 2014 by author and the journal "Automation technological and business - processes". This work is licensed under the Creative Commons Attribution International License (CC BY). http://creativecommons.org/licenses/by/4.0/

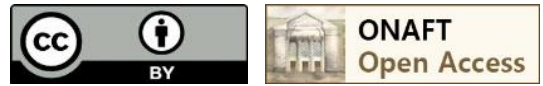

DOI: $10.15673 / 2312-3125$.

\section{Аннотация}

Проведен анализ существующх принципов частотного управления и обоснован выбор скалярного принципа для управления трехфазным асинхронным электродвигателем компрессора. Определены регламентированные значения пульсаций частоты вращения электродвигателя компрессора при различных диапазонах регулирования скорости и на основе этого предложено использование замкнутой двухконтурной системы подчиненного регулирования координат при работе преобразователя частоты в режиме источника тока в качестве системы частотного управления. Предложен эффективный бездатчиковый способ определения частоты вращения и момента сопротивления электродвигателя компрессора на основе теории наблюдателей Люенбергера. Определены размерность и коэффициенты матрицы Люенбергера, исходя из равенства величины среднегеометрического корня характеристического полинома системы управления значению угловой частоты напряжения сети. Проведено моделирование полученной системы частотного управления электродвигателем компрессора в среде визуального программирования Matlab/Simulink на примере трехфазного асинхронного двигателя 4АA63А4У3. Проанализировав данные моделирования, было выявлено, что данная система управления не обеспечивает необходимой жесткости механических характеристик в динамике, поскольку пульсации частоты вращения 


\section{2 АВТОМАТИЧНІ ТА АВТОМАТИЗОВАНІ СИСТЕМИ УПРАВЛІННЯ ТЕХНОЛОГІЧНИМИ ПРОЦЕСАМИ}

электродвигателя компрессора не соответствуют регламентированным требованиям. Для устранения данного недостатка синтезирован корректирующий канал воздействия по моменту сопротивления на валу двигателя для повышения робастности системы. Определены пульсации частоты вращения электропривода компрессора при различных сигналах задания скорости. Доказано, что введение в систему управления положительной обратной связи через корректирующее звено снижает пульсации частоты вращения до регламентированных значений.

\section{Abstract}

The analysis of the existing principles of frequency conrol is carried out and the choice of the scalar principle for the compressor three-phase asynchronous electric motor control is justified. The regulated values of the rotation frequency pulsations of the compressor electric motor at various ranges of the speed regulation are defined and on the basis of it use of the closed double-circuit system of the coordinates slave regulation during the operation of the frequency converter in the current source mode as a system of the frequency control is offered. The effective sensorless metod of the rotation frequency and the resistance torque determination of the compressor electric motor on the basis of the Lyuenberger observers theory is offered. Dimension and coefficients of a Lyuenberger matrix proceeding from equality of the root geometric mean value of the control system characteristic polynomial and the angular frequency of the supply voltage are defined. Modeling of the received frequency control system of the compressor electric motor in the environment of visual programming Matlab/Simulink on the example of the threephase asynchronous engine 4AA63A4U3 is carried out. Having analysed data of modeling, it was revealed that this control system doesn't provide necessary rigidity of the mechanical characteristics in dynamics as rotation frequency pulsations of the compressor electric motor don't conform to the regulated requirements. For elimination of this disadvantage the correcting channel of influence on resistance torque on the motor for increase of the system robastness is synthesized. Rotation frequency pulsations of the compressor electric drive at various signals of the speed task are defined. It is proved that introduction the positive feedback through the corrective element to the control system reduces rotation frequency pulsations to the regulated values.

Ключевые слова

Компрессор, трехфазный асинхронный двигатель, электропривод, частотное управление, наблюдатель Люенбергера.

Постановка проблемы

В настоящее время многие производители холодильной техники расширяют количество продукции, использующей регулируемый электропривод компрессора. Это холодильные установки рефрижераторных судов, винные шкафы, холодильные камеры для хранения плазмы крови и т.д. В основном такая продукция носит узкоспециализированный характер, имеет небольшие объемы выпуска, мощность компрессоров превышает значение 1000 Вт. Единственным массовым представителем холодильных техники с регулируемым электроприводом являются кондиционеры инверторного типа. Указанные примеры холодильной техники составляют всего до $10 \%$ сегмента холодильного оборудования, производимого в мире и Украине. Использование в них регулируемого электропривода обусловлено требованиями к точности поддержания температуры в холодильной камере.

Остальная большая часть производимой холодильной продукции работает в двухпозиционном режиме регулирования температуры. Это сегмент торгового холодильного оборудования (ТХО), который включает в себя бытовые холодильные приборы, малые холодильные камеры, морозильные камеры, холодильные витрины, холодильные торговые автоматы холодопроизводительностью не более 1000 Вт. Внедрение регулируемого электропривода в ТХО считается экономически нецелесообразным и исследований в этом направлении [2,3] крайне мало, и они носят в основном описательный характер.

Для комплексной оценки целесообразности перехода к регулируемому электроприводу в сегменте ТХО необходимо учитывать большой объем выпуска продукции (свыше 1 млн. единиц в год). Повышение КПД или уменьшение материалоемкости ТХО даже на доли процента может дать ежегодную прибыль в миллионы гривен. В работе [1] была показана возможность снижения габаритных размеров и массы асинхронных двигателей герметичных компрессоров малых холодильных установок (МХУ) при переходе от традиционной системы управления холодопроизводительностью к использованию системы «преобразователь частоты - трехфазный асинхронный двигатель» (ПЧ-ТАД). Такое техническое решение позволит существенно сократить затраты на 


\section{2 АВТОМАТИЧНІ ТА АВТОМАТИЗОВАНІ СИСТЕМИ УПРАВЛІННЯ ТЕХНОЛОГІЧНИМИ ПРОЦЕСАМИ}

изготовление электропривода компрессора, однако при этом остаются неизученными проблемы эффективного управления модернизированными двигателями компрессоров [1]. Целью данного исследования является создание эффективной системы частотного управления ТАД герметичных компрессоров МХУ.

Для достижения поставленной цели необходимо решить ряд частных задач:

- п произвести выбор простой и экономичной системы частотного управления, обеспечивающей необходимые динамические показатели качества процесса регулирования скорости вращения ТАД компрессора;

- обеспечить необходимый диапазон регулирования скорости при допустимых пульсациях скорости.

Как известно из [3], для нормальной работы герметичного компрессора МХУ при частотном управлении достаточно обеспечить диапазон регулирования скорости 1:3. Также, учитывая требования к простоте системы частотного управления, можно сделать вывод о целесообразности выбора скалярного принципа частотного управления, поскольку он является технически наиболее простым и обеспечивает необходимый диапазон регулирования [4].

Построение скалярного принципа управления возможно при работе преобразователя частоты в режиме источника напряжения и в режиме источника тока [4]. При работе ПЧ в режиме источника напряжения возможно построение наиболее простой разомкнутой системы управления, но ее динамические показатели и жесткость механических характеристик электропривода даже в замкнутом состоянии будут невелики. Работа ПЧ в режиме источника тока позволяет получить более жесткие механические характеристики электропривода с сохранением постоянства критического момента, однако она более сложна и в разомкнутом состоянии будет практически неработоспособной.

Для окончательного выбора системы управления необходимо учитывать характер изменения нагрузки на валу двигателя компрессора и требования к допустимым пульсациям скорости вращения. Как показано в [5], момент сопротивления на валу двигателя поршневого компрессора имеет резко выраженный пульсирующий характер, причем максимальная его величина за один оборот вала двигателя может достигать трехкратного превышения номинального значения. Такие пульсации момента сопротивления приводят к пульсациям скорости вращения коленчатого вала, на который насажен ротор двигателя [5]. Неравномерность вращения коленчатого вала компрессора характеризуется степенью неравномерности вращения (пульсациями частоты вращения):

$$
\delta=\frac{\omega_{\max }-\omega_{\min }}{\omega_{c p}}
$$

где $\omega_{\max }, \omega_{\min }$ и $\omega_{c p}$ - максимальная, минимальная и средняя скорость соответственно. Для асинхронного двигателя, насаженного на вал компрессора, при номинальной скорости вращения регламентируется значение $\delta<1 / 80$. Такое значение $\delta$ обусловлено допустимой тепловой нагрузкой обмоток двигателя. Поскольку большая часть тепловой мощности, выделяемой в статоре двигателя, обусловлена магнитными потерями, пропорциональными квадрату частоты питающего напряжения, то при частотном управлении степень неравномерности $\delta$ может увеличиваться обратно пропорционально квадрату диапазона регулирования скорости (

таблица 1).

Таблица 1 - Зависимость степени неравномерности $\delta$ от скорости вращения $\omega$

\begin{tabular}{|c|c|c|c|c|}
\hline$\omega$ & $\omega_{\text {ном }}$ & $1 / 2 \cdot \omega_{\text {ном }}$ & $1 / 3 \cdot \omega_{\text {ном }}$ & $1 / 4 \cdot \omega_{\text {ном }}$ \\
\hline$\delta, \%$ & 1,25 & 5 & 11,25 & 20 \\
\hline
\end{tabular}

С учетом изложенных требований к величине пульсаций скорости вращения использование разомкнутых систем управления нецелесообразно по причине малой жесткости механических характеристик. Максимальную жесткость механических характеристик можно получить в замкнутой двухконтурной системе подчиненного регулирования координат при работе преобразователя в режиме источника тока [4]. Эту систему и будем использовать в качестве исходной.

Построение системы подчиненного регулирования координат электропривода с регуляторами тока и скорости невозможно без наличия датчика скорости двигателя. Установка датчика скорости в герметичный компрессор сопряжено с большими техническими сложностями и является нецелесообразным. Существующие системы бездатчикового определения скорости [6] требуют относительных сложных координатных преобразований векторов измеряемых величин и выполнения операции дифференцирования. Во избежание усложнения системы управления авторами предлагается использовать неизученный ранее способ бездатчикового определения скорости двигателя, основанный на использовании наблюдателей Люенбергера. 


\section{2 АВТОМАТИЧНІ ТА АВТОМАТИЗОВАНІ СИСТЕМИ УПРАВЛІННЯ ТЕХНОЛОГІЧНИМИ ПРОЦЕСАМИ}

Основные элементы предлагаемой системы управления электроприводом компрессора показаны на рис. 1. Здесь обозначено: ЗИ - задатчик интенсивности, РС - регулятор скорости, ФП - функциональный преобразователь, РТ - регулятор тока статора, Ф - фильтр, ИМ - имитационная модель двигателя, ЭММ электромагнитная часть модели двигателя, $L$ - матрица Люенбергера, ДТ - датчик тока, ДН - датчик напряжения, ВМТ - вычислитель момента и вектора тока двигателя, $K c$ - коэффициент обратной связи по скорости, Uзснапряжение задания скорости, $U p c$ - напряжение регулятора скорости, $U з m$ - напряжение задания тока, $U p m-$ напряжение регулятора тока, Uзч - напряжение задания частоты, Uос - напряжение обратной связи по скорости, $U o m$ - напряжение обратной связи по току, $M$ - сигнал, пропорциональный моменту двигателя; $\hat{M}, \hat{M}_{C}, \hat{\omega}-$ оцененные значения электромагнитного момента, момента сопротивления и скорости ТАД компрессора соответственно. Назначение элементов РС, ФП, РТ, ДТ и ДН описывается в [4]. В качестве ЭММ можно использовать модель ТАД, приведенную в [6].

Имитационная модель ТАД компрессора (рис. 1) моделирует работу двигателя на холостом ходу без учета момента сопротивления. Для идентификации скорости ТАД, работающего под нагрузкой, в модель введена коррекция, представляющая собой одну из форм известного наблюдателя Люенбергера [7]. Как видно из рис. 1, сигналом коррекции служит разность момента ТАД, вычисляемого блоком ВМТ и оценки момента на выходе блока ЭММ. Полученная разность матрицей $L$ преобразуется в два корректирующих сигнала, один из которых при выбранной топологии имитационной модели ТАД представляет собой оценку момента сопротивления:

$$
\widehat{M} c=L_{1} \cdot(M-\bar{M})
$$

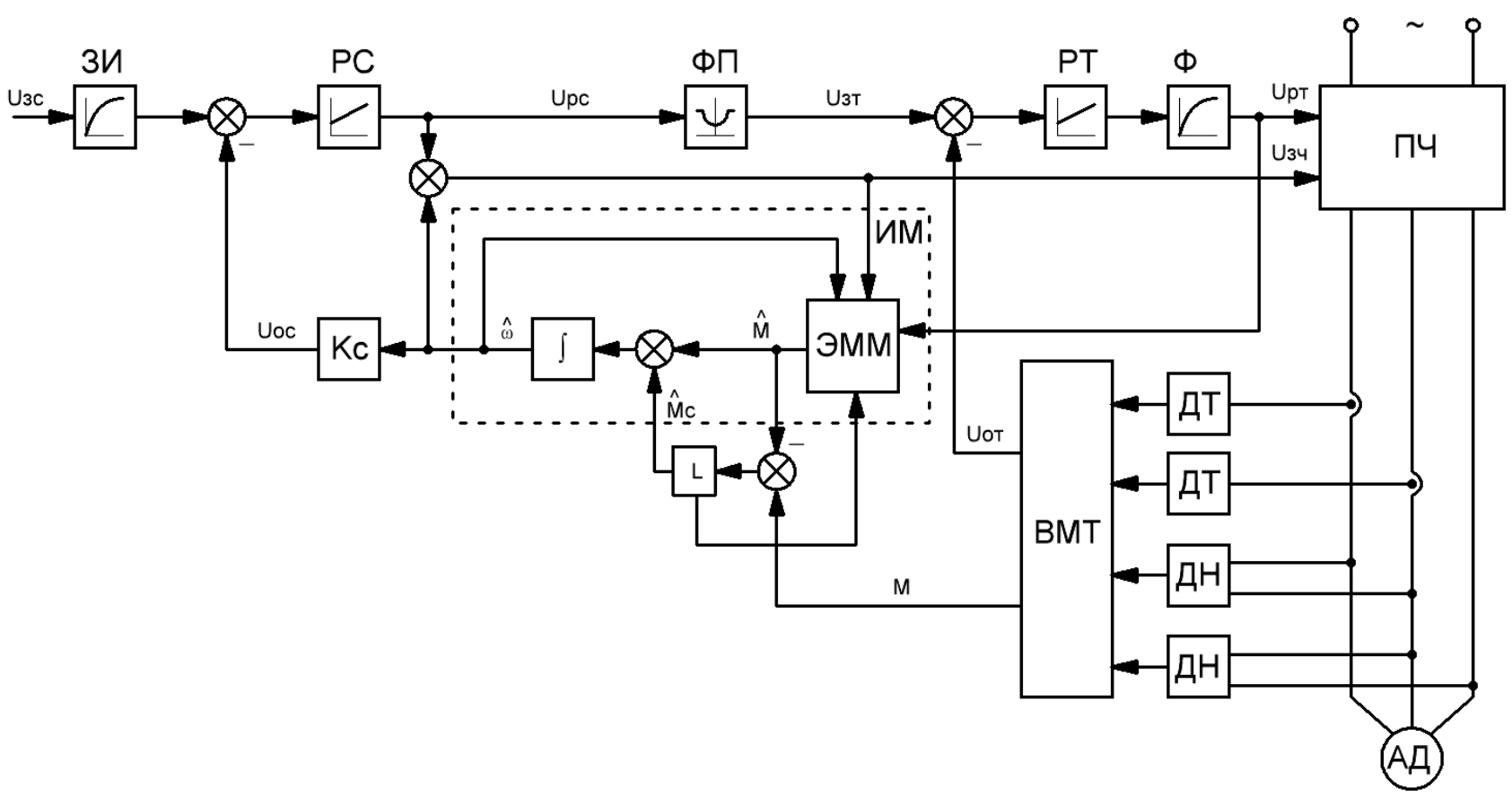

Рис. 1. Структурная схема системы управления электроприводом компрессора с использованием модели двигателя

Определение размерности и коэффициентов матрицы Люенбергера $L$ проводилось по методике, изложенной в [7]. Предварительно были идентифицированы параметры схемы замещения модернизированных ТАД типа 4АА63А4У3, используя данные из [1]. Поскольку линеаризованная модель ТАД представляет собой объект управления второго порядка, и из всех величин состояния известной является только вычисленное значение электромагнитного момента $M$, то возможным является реализация наблюдателя Люенбергера второго порядка [7]. В таком случае матрица $L$ будет иметь размер $1 \times 2$. Значение коэффициентов матрицы $L$ в общем случае определяется необходимым быстродействием наблюдателя, которое в $5 \ldots 10$ раз должно превышать быстродействие процессов, протекающих в ТАД. Для количественной оценки желаемого быстродействия используют величину среднегеометрического корня $\Omega$ характеристического полинома системы управления [7]. Так 


\section{2 АВТОМАТИЧНІ ТА АВТОМАТИЗОВАНІ СИСТЕМИ УПРАВЛІННЯ ТЕХНОЛОГІЧНИМИ ПРОЦЕСАМИ}

как скорость всех динамических процессов, протекающих в ТАД, определяется в первую очередь номинальной частотой напряжения, подводимого к двигателю, то рекомендуется принять значение среднегеометрического корня равным

$$
\Omega=\omega_{0}=628 \frac{\text { рад }}{\mathrm{c}}
$$

где $\omega_{0}-$ номинальная угловая частота напряжения модернизированных ТАД.

С учетом изложенных рекомендаций и найденных параметров исследуемых ТАД в схеме на рис. 1 были рассчитаны параметры настройки регуляторов тока и скорости, постоянные времени датчиков тока, напряжения и фильтров и определены величины коэффициентов матрицы $L$. Моделирование схемы проводилось в среде моделирования Matlab/Simulink. В результате моделирования были получены графики пуска ТАД 4АА63А4У3 при различных управляющих воздействиях (рис. 2a).
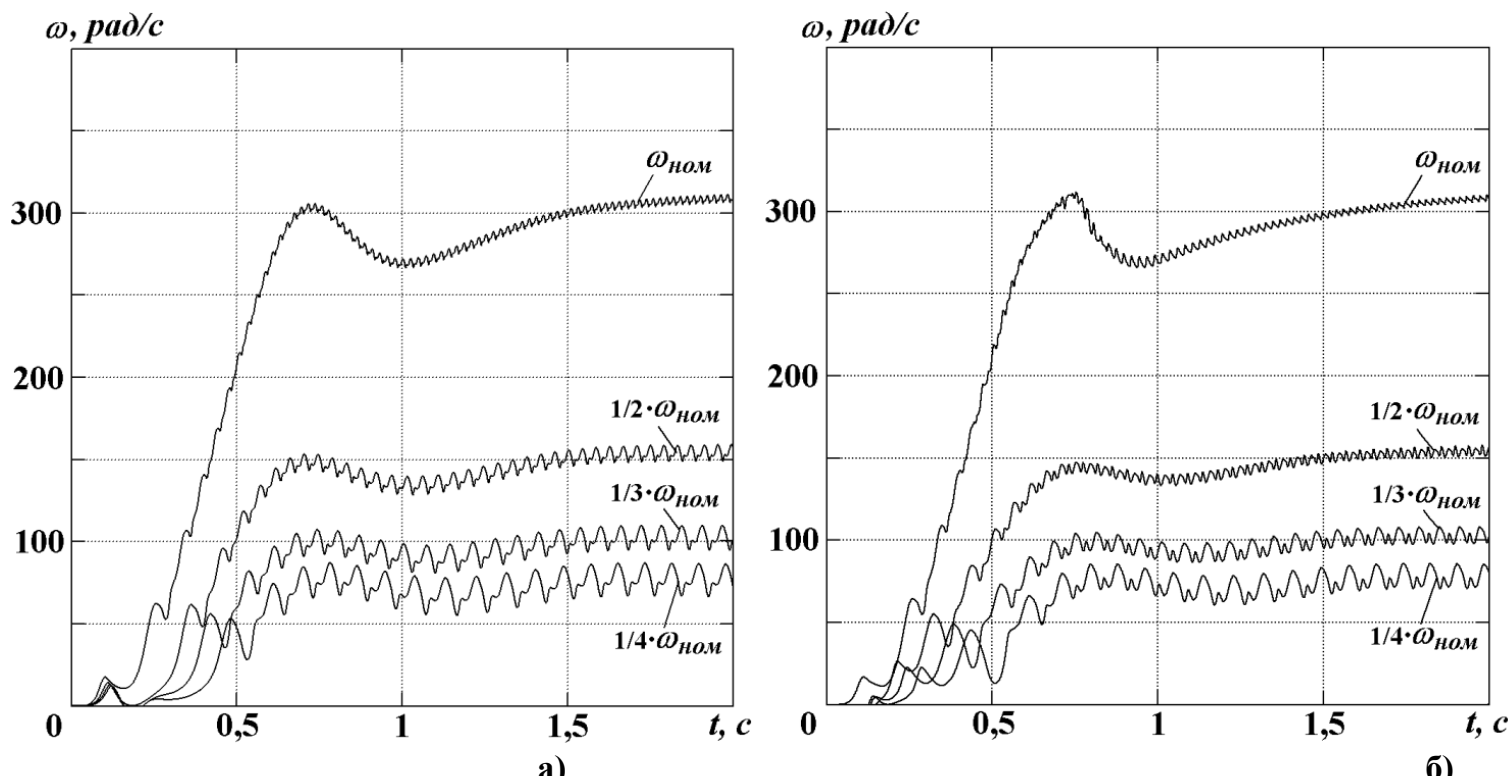

Рис. 2. Графики пуска ТАД компрессора 4АА63А4У3:

б)

а) без корректирующего звена; б) с корректирующим звеном

Проанализировав данные моделирования, было выявлено, что данная система управления не обеспечивает необходимой жесткости механических характеристик в динамике, поскольку пульсации скорости при всех четырех сигналах задания не соответствуют требованиям, приведенным в

таблица 1. Такое несоответствие связано с сильно уменьшенным по сравнению с аналогичными однофазными двигателями моментом инерции исследуемых ТАД. Для решения выявленной проблемы предлагается использовать корректирующий канал воздействия по моменту сопротивления двигателя, оценка которого формируется синтезированным наблюдателем Люенбергера на выходе блока $L$ (рис. 1). Синтез корректирующего звена проводился методом обратных передаточных функций на основе линеаризованной модели системы частотного управления ТАД с регулятором скорости, приведенной в [4]. В результате получена адаптивная система управления электроприводом герметичного компрессора со свойствами робастности, представленная на рис. 3.

Из рис. 3 можно увидеть, что полученное корректирующее звено (КЗ) определяется параметрами исследуемого ТАД: электромагнитной постоянной времени ротора $T_{2}$ и моментом инерции $J$ и коэффициентом обратной связи по скорости $K c$. Наличие идеального дифференцирующего звена в составе КЗ требует установки перед ним фильтра с постоянной времени на $1 . .2$ порядка меньшей $T_{2}$. В результате моделирования предложенной системы управления были получены графики пуска ТАД 4АА63А4У3, представленные на рис. 2б. Сравнивая графики на рис. $2 \mathrm{a}$ и рис. 2б, можно визуально отметить снижение пульсаций скорости при использовании КЗ. Для количественной оценки преимуществ системы с КЗ перед исходной схемой были определены пульсации скорости (коэффициенты 


\section{$\underline{2}$ АВТОМАТИЧНІ ТА АВТОМАТИЗОВАНІ СИСТЕМИ УПРАВЛІННЯ ТЕХНОЛОГІЧНИМИ ПРОЦЕСАМИ}

неравномерности вращения) для приведенных схем при четырех значениях сигнала задания скорости, соответствующих четырем диапазонам регулирования. Результаты расчетов приведены в таблица 2.

В результате проведенного исследования с учетом данных таблица 2 можно сделать следующие выводы:

1. Для управления ТАД герметичных компрессоров МХУ целесообразно использовать скалярный принцип управления при работе ПЧ в режиме управляемого источника тока.

2. При необходимости подавления пульсаций скорости ТАД компрессора возможно использовать предложенную адаптивную систему управления с элементами робастности. Пульсации скорости при введении в схему корректирующего звена удовлетворяют регламентированным значениям.

3. Для определения в реальном времени скорости и момента сопротивления ТАД компрессора предложен эффективный бездатчиковый способ, заключающийся в использовании принципов построения наблюдателя Люенбергера второго порядка. Результаты моделирования замкнутой системы управления доказывают работоспособность предложенного способа.

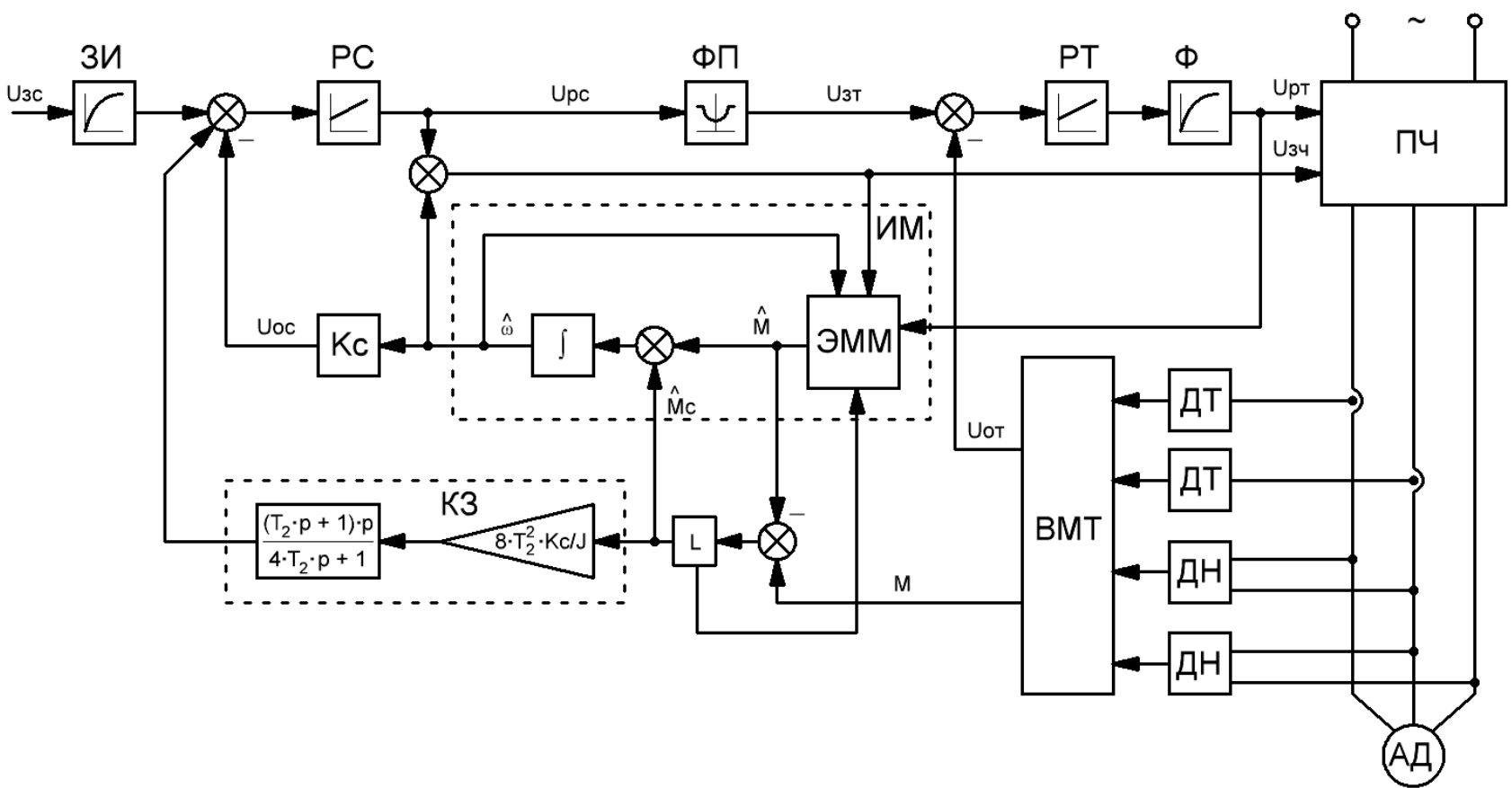

Рис. 3. Структурная схема адаптивной системы управления електроприводом компрессора со свойствами робастности

Таблица 2 - Пульсации скорости при различных сигналах задания скорости

\begin{tabular}{|c|c|c|c|}
\hline \multirow{2}{*}{$\begin{array}{l}\text { Сигнал задания } \\
\text { скорости } U з c, \text { В }\end{array}$} & \multirow{2}{*}{$\begin{array}{c}\text { Диапазон } \\
\text { регулирования }\end{array}$} & \multicolumn{2}{|c|}{ Пульсации скорости $\delta, \%$} \\
\hline & & $\begin{array}{c}\text { Система без КЗ } \\
\text { (рис. 1) }\end{array}$ & $\begin{array}{c}\text { Система с К3 } \\
\text { (рис. 3) }\end{array}$ \\
\hline 10 & $1: 1$ & 1,5 & 1,1 \\
\hline 5 & $1: 2$ & 6,1 & 4,1 \\
\hline 3,33 & $1: 3$ & 13,8 & 9,5 \\
\hline 2,5 & $1: 4$ & 25,3 & 19,3 \\
\hline
\end{tabular}

\section{Литература}

1. Букарос А.Ю. Модернизация управляемых приводов герметичных компрессоров / А.Ю. Букарос, О.А. Онищенко // Електротехнічні та комп'ютерні системи. - 2010. - № 01 (77). - С. 58-63.;

2. Войтех В.А. Частотное регулирование скорости вращения асинхронних двигателей компрессоров бытовых холодильников / Войтех В.А. // Техн. електродинаміка. Темат. вип. "Проблеми сучасної електротехніки”. 2004. - Ч. 3. - С. 61-63.; 


\section{2 АВТОМАТИЧНІ ТА АВТОМАТИЗОВАНІ СИСТЕМИ УПРАВЛІННЯ ТЕХНОЛОГІЧНИМИ ПРОЦЕСАМИ}

3. Онищенко О.А. Система управления электроприводом поршневого компрессора холодильной установки / Онищенко О.А. // Електромашинобудування та електрообладнання. - 2005. - С. 23-28.;

4. Терехов В.М. Системы управления электроприводов: учебник / В.М. Терехов, О.И. Осипов - М.: «Академия», 2005. - 301 с.

1. Пластинин П.И. Поршневые компрессоры. Том 1. Теория и расчет. / Пластинин П.И. - М.: Колос, 2006. $456 \mathrm{c}$.

2. Соколовский Г.Г. Электроприводы переменного тока с частотным регулированием: учебник / Соколовский Г.Г. - М.: «Академия», 2006. - 266 с.

3. Панкратов В.В. Избранные разделы теории автоматического управления: учебное пособие / В.В Панкратов, О.В. Нос, Е.А. Зима - Новосибирск: Издательство НГТУ, 2011. - 223 с.

References

1. Bukaros A.Yu. Modernizatsiya upravlyaemyih privodov germetichnyih kompressorov / A.Yu. Bukaros, O.A. Onischenko // ElektrotehnIchnI ta komp'yuterni sistemi. - 2010. - \# 01 (77). - P. 58-63.;

2. Voyteh V.A. Chastotnoe regulirovanie skorosti vrascheniya asinhronnih dvigateley kompressorov byitovyih holodilnikov / Voyteh V.A. // Tehn. elektrodinamika. Temat. vip. "Problemi suchasnoyi elektrotehniki". - 2004. P. 3. - P. 61-63.;

3. Onischenko O.A. Sistema upravleniya elektroprivodom porshnevogo kompressora holodilnoy ustanovki / Onischenko O.A. // Elektromashinobuduvannya ta elektroobladnannya. - 2005. - P. 23-28.;

4. Terehov V.M. Sistemyi upravleniya elektroprivodov: uchebnik / V.M. Terehov, O.I. Osipov - M.: «Akademiya», 2005. - 301 p.;

5. Plastinin P.I. Porshnevyie kompressoryi. Tom 1. Teoriya i raschet. / Plastinin P.I. - M.: Kolos, $2006 .-456$ s.;

6. Sokolovskiy G.G. Elektroprivodyi peremennogo toka s chastotnyim regulirovaniem: uchebnik / Sokolovskiy G.G. - M.: «Akademiya», 2006. - 266 p.;

7. Pankratov V.V. Izbrannyie razdelyi teorii avtomaticheskogo upravleniya: uchebnoe posobie / V.V. Pankratov O.V. Nos, E.A. Zima - Novosibirsk: Izdatelstvo NGTU, 2011. - 223 p.

\section{АНАЛИЗ ЧУВСТВИТЕЛЬНОСТИ ЦЕЛЕВОЙ ФУНКЦИИ ДЛЯ УПРАВЛЕНИЯ СИСТЕМАМИ ТЕПЛОСНАБЖЕНИЯ ГОРОДСКИХ РАЙОНОВ}

Бабич С.B. ${ }^{1}$

${ }^{1}$ Одесский национальный политехнический университет, Одесса

Copyright (C) 2014 by author and the journal "Automation technological and business - processes". This work is licensed under the Creative Commons Attribution International License (CC BY). http://creativecommons.org/licenses/by/4.0/

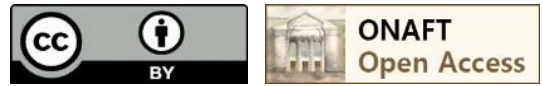

DOI: $10.15673 / 2312-3125$.

\section{Аннотация}

Процесс теплоснабжения современного Украинского города должен соответствовать высоким требования надежности и эффективности. Как правило задачи отопления и горячего водоснабжения решаются централизовано сетью районных котельных. В тоже время износ оборудования и магистральных трубопроводов приводит к росту тепловых и физических потерь теплоносителя, что существенно снижает эффективность процесса теплоснабжения. Это приводит к тому, что часть потребителей города переходит на альтернативные источники тепла такие как: крышные и модульные котельные, индивидуальные 